\title{
Exploiting Mobility in Proportional Fair Cellular Scheduling: Measurements and Algorithms
}

\author{
Robert Margolies*, Ashwin Sridharan ${ }^{\dagger}$, Vaneet Aggarwal ${ }^{\dagger}$, \\ Rittwik Jana $^{\dagger}$, N. K. Shankaranarayanan ${ }^{\dagger}$, Vinay A. Vaishampayan ${ }^{\dagger}$, Gil Zussman* \\ ${ }^{*}$ Electrical Engineering, Columbia University, New York, NY $10027{ }^{\dagger}$ AT\&T Labs - Research, NJ \\ $\{$ robm,gil\}@ee.columbia.edu, $\{$ asridharan, vaneet, rjana, shankar, vinay\}@ research.att.com
}

\begin{abstract}
Proportional Fair (PF) scheduling algorithms are the de-facto standard in cellular networks. They exploit the users' channel state diversity (induced by fast-fading), and are optimal for stationary channel state distributions and an infinite time-horizon. However, mobile users experience a non-stationary channel, due to slow-fading (on the order of seconds), and are associated with basestations for short periods. Hence, we develop the Predictive Finite-horizon PF Scheduling $\left((P F)^{2} S\right)$ Framework that exploits mobility. We present extensive channel measurement results from a 3G network and characterize mobility-induced channel state trends. We show that a user's channel state is highly reproducible and leverage that to develop a data rate prediction mechanism. We then present a few channel allocation estimation algorithms that rely on the prediction mechanism. Our trace-based simulations consider instances of the $\mathbf{P F}^{2} \mathbf{S}$ Framework composed of combinations of prediction and channel allocation estimation algorithms. They indicate that the framework can increase the throughput by $15 \%-55 \%$ compared to traditional PF schedulers, while improving fairness.
\end{abstract}

Keywords-Cellular networks, Mobility, Proportional fairness, Measurements, Channel state prediction, Slow-fading.

\section{INTRODUCTION}

3G and 4G (LTE) cellular networks incorporate opportunistic schedulers [9]. These schedulers allocate resources to users with good channel conditions by leveraging channel state variations, due to fast-fading, ${ }^{1}$ as well as multi-user diversity. Proportional Fair (PF) scheduling algorithms are the defacto standard for opportunistic schedulers in cellular networks [13]. They aim to provide high throughput while maintaining fairness among the users. PF scheduling algorithms have been extensively studied in the past (e.g., [5], [8], [15]). These algorithms are optimal under the assumptions that the wireless channel state is a stationary process (i.e., it is subject only to fast-fading) and the users' association times are long (e.g., static users or pedestrians) [17], [25]. However, when these assumptions do not hold (which is the case for mobile users), the performance of these algorithms is suboptimal [4].

For example, Fig. 1 illustrates a trajectory of a car along a $5 \mathrm{~km}$ path, and the signal quality $\left(E_{c} / I_{o}\right)$ to 3 different sectors (we collected the $E_{c} / I_{o}$ values during 3 drives on the path). As can be seen, the channel has a dominant slow-fading component $t^{2}$ on which the fast-fading component is overlaid. Since

\footnotetext{
${ }^{1}$ Fast-fading is characterized by rapid fluctuations in the received signal strength (due mainly to multipath) [22].

${ }^{2}$ Slow-fading is characterized by slow (on the order of seconds) changes of the received signal strength (e.g., due to path loss and shadowing) [22].

978-1-4799-3360-0/14/\$31.00 (c)2014 IEEE
}
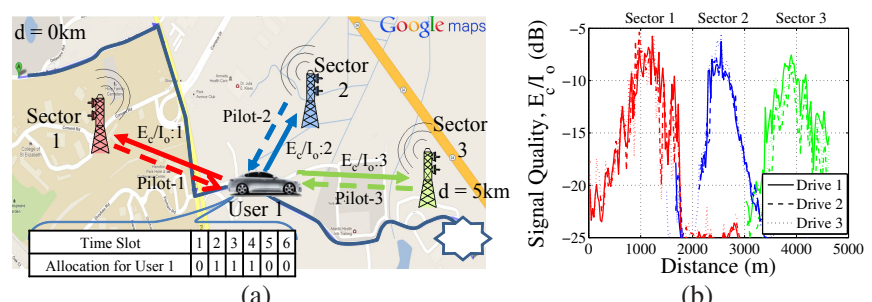

(b)

Fig. 1. Mobile user trajectory along a road through 3 cellular sectors: (a) map outline and interactions with the cellular network and (b) measured values of channel quality $\left(E_{c} / I_{o}\right)$ during 3 different drives.

$E_{c} / I_{o}$ has noticeable trends over several seconds, the channel state distribution is non-stationary. Additionally, movement along the path initiates hand-offs between the sectors, and therefore, the association periods are short.

Since PF schedulers are not optimized for mobility, we design the Predictive Finite-horizon PF Scheduling ( $\left.(\mathrm{PF})^{2} \mathrm{~S}\right)$ Framework which is tailored for mobile nodes and that takes advantage of both slow- and fast-fading. It includes three components: (i) data rate prediction, (ii) estimation of future channel allocations, and (iii) slow-fading aware scheduling.

To characterize slow-fading, to provide input to the design of the rate prediction mechanism, and to obtain traces for the evaluation of the framework and algorithms, we conducted an extensive measurement campaign. In particular, we discuss fine-grained (i.e., millisecond resolution) measurements, collected from a $3 \mathrm{G}$ network. ${ }^{3}$ Specifically, we measured wireless channel attributes in drives spanning $810 \mathrm{~km}$ and during a period of over 1,300 minutes. Unlike a few previous studies (e.g., [24]) which measured the Received Signal Strength Indicator (RSSI) which is the total received power in a frequency band, we measured the signal quality to each sector $\left(E_{c} / I_{o}\right)$. This allows us to obtain important insights, since $E_{c} / I_{o}$ is the most relevant predictor of a user's data rate.

We analyze the traces and show that mobile users experience pronounced slow-fading. However, the slow-fading trends cannot be simply tied to line-of-sight metrics, and therefore, developing simple channel state predictions is infeasible. Yet, the slow-fading component of $E_{c} / I_{o}$ is remarkably reproducible for multiple drives on the same path (e.g., Fig. 1(b)), lending itself to data-driven prediction approaches.

Based on these observations, we develop a 2-phase rate prediction mechanism (referred to as the Coverage Map Prediction

\footnotetext{
${ }^{3}$ The measurements were collected from a $3 \mathrm{G}$ network, due to lack of ubiquity of LTE networks. Yet, our observations regarding slow-fading apply to $4 \mathrm{G}$ networks, as they operate at similar time-scales.
} 
Mechanism (CMPM)). In an offline phase, measurement traces are processed to construct channel quality maps. The online phase is conducted by the sector and includes determination of the user's location and velocity, and thereby the predicted data rate. The localization can be simply done by querying the user's GPS. However, since this imposes energy and computation burdens on the user, we also develop the Channel History Localization Scheme (CHLS) which requires some knowledge of the user's trajectory. ${ }^{4}$ CHLS uses a variation of the Dynamic Time Warping (DTW) algorithm (originally developed for speech recognition [21]).

The $(\mathrm{PF})^{2} \mathrm{~S}$ Framework also requires algorithms that estimate the future channel allocations based on the rate predictions. We propose three such heuristic algorithms with different degrees of robustness to prediction errors and different performance levels for relatively accurate predictions. Using test cases generated from the collected traces, we perform an extensive simulation evaluation of the $(\mathrm{PF})^{2} \mathrm{~S}$ Framework. We consider 9 framework instances, representing combinations of rate prediction mechanisms and channel allocation estimation algorithms. We show that various instances of the framework consistently outperform the PF scheduler. Specifically, throughput improvements in realistic mobile scenarios range from $15 \%$ to $55 \%$ (with maintained or improved fairness levels). Finally, we study the sensitivity of the framework and algorithms to various network parameters and assumptions, including number of users and delay constraints.

The main contributions of this paper are 3-fold: (i) it demonstrates, based on an extensive measurement campaign, that mobile users experience a reproducible but non-stationary slow-fading channel; (ii) it provides a cellular scheduling framework (and corresponding algorithms), tailored for mobile users; and (iii) it shows (using trace-based simulations) that the framework can significantly improve performance.

The paper is organized as follows. Section II discusses related work and Section III reviews channel state metrics and formulates the problem. Section IV presents the scheduling framework. Section V discusses the measurements and characterization of slow-fading. A rate prediction mechanism is presented in Section VI and algorithms to estimate future channel allocations are presented in Section VII. The framework and algorithms are evaluated in Section VIII. We conclude and discuss future work in Section IX. Due to space constraints, several simulation results are omitted and can be found in [20].

\section{RELATED WORK}

Opportunistic Scheduling: As mentioned, opportunistic and PF scheduling have been extensively studied (e.g., [5], [9], [15], [17], [25]). PF scheduling algorithms using fast-fading channel state predictions appear in [6], [11] (without a prediction mechanism). Scheduling for mobile users is considered in [2], [8], [24], where the underlying assumption in [2], [8] is that the user's mobility patterns induce a stationary (and known) slow-fading channel. The algorithm of [24] schedules a single user using an RSSI-based prediction method at time scales on the order of minutes. On the other hand, we solve a multi-user scheduling problem at finer time scales (tens of seconds) using an $E_{c} / I_{o}$-based prediction mechanism.

\footnotetext{
${ }^{4}$ As such, it is highly applicable to users on highways and major roads.
}

Channel Measurements and Predictions: Wireless channel measurement studies have been conducted for decades [3], [12]. Recently, [18] studied the interaction of applications and the physical layer attributes in the 1x-EVDO network (using a predecessor to our measurement tool). Slow-fading is studied in controlled environments in [26]. Methods for short-term (over a few milliseconds) prediction of non-stationary wireless channel states appear in [19]. The measurements in [27], [28] focus on the repeatability of achieved bandwidth in a $3 \mathrm{G}$ network. Unlike previous works, we conduct measurements of wireless channel quality in a $3 \mathrm{G}$ network to characterize and predict slow-fading patterns over tens of seconds.

Localization and Mobility Prediction: Localization in cellular networks includes approaches that utilize time of arrival, time-difference of arrival, angle-of-arrival, cell-ID, and received signal strength (see [14] and references therein). Mobility prediction schemes that utilize pattern tracking and learning algorithm are reviewed in [16]. The method in [10] uses the DTW algorithm, albeit for velocity estimation. The closest related works are [14], [24] that utilize RSSI in GSM networks to localize users via fingerprinting. On the other hand, our scheme uses multiple channel attributes (i.e., $E_{c} / I_{o}$ and RSSI) as well as recent history and is evaluated via tracebased simulations.

\section{MOdEL AND PROBLEM Formulation}

\section{A. Channel States in $3 G$ Networks}

In a $3 \mathrm{G}$ network [13], each basestation covers a cell which is divided into (typically 3) sectors. As illustrated in Fig. 1(a), for data scheduling and hand-off purposes, users estimate the wireless channel quality to each nearby sector. It is estimated as the ratio between the power of a sector-specific pilot signal and the total in-band power (including interference and noise), and is denoted by $E_{c} / I_{o}$. In Section $\mathrm{V}$, we will consider these values in our measurement study.

A user associates (connects) with the strongest neighboring sector, termed the serving sector, and is assigned a dedicated buffer at the sector. When the serving sector $E_{c} / I_{o}$ value drops below a threshold (e.g., due to mobility), the user hands-off wherein it disassociates from the serving sector and connects to a new sector with a higher $E_{c} / I_{o}$ value.

The downlink channel from the sector to the users is timeslotted. We will denote by $E_{c} / I_{o}[j]$ the value in time slot $j$. The users periodically report their $E_{c} / I_{o}$ to the sector. Then, an appropriate channelization code is selected and mapped to a feasible data rate. ${ }^{5}$ The feasible data rate of user $i$ in slot $j$ is denoted $r_{i j}$. An opportunistic scheduler implemented in the sector utilizes the multiuser diversity of the data rates to allocate downlink slots to users (see Fig. 1(a)). ${ }^{6}$

\section{B. Scheduling Problem Formulation}

The common 3G scheduler solves a Proportional Fair (PF) Scheduling Problem [13], [15] and aims to achieve high overall throughput while maintaining fairness among the users. The

\footnotetext{
${ }^{5}$ The mapping from $E_{c} / I_{o}$ to data rates is described in [20]. The mapping is phone specific and for our phones, the maximum data rate is $20 \mathrm{Mbps}$.

${ }^{6}$ Multiple users (typically, no more than 4) may share a slot. Practically, it is uncommon, and we assume that exactly one user is allocated a slot.
} 
common assumptions regarding stationary channels and long association times do not hold in mobile scenarios (as will be shown in Section V). Hence, we formulate the downlink scheduling problem as a variant of the PF Scheduling Problem while utilizing a formulation similar to [4] (which studied adversarial channels). Unlike previous work, (e.g., [8], [17], [25]), we do not make assumptions regarding the channel state distributions and optimize over a finite time horizon.

We assume that a sector has $K$ associated users with backlogged downlink buffers. ${ }^{7}$ Denote by $\alpha_{i j}$ the scheduler allocation $\left(\alpha_{i j}=1\right.$, if user $i$ is allocated slot $j$, and $\alpha_{i j}=0$, otherwise). We denote the feasible data rate and the scheduler allocation matrices by $\mathbf{R}=\left\{r_{i j}\right\}_{K \times T}$ and $\boldsymbol{\alpha}=\left\{\alpha_{i j}\right\}_{K \times T}$, respectively. We assume a finite time horizon of $T$ slots that corresponds to the users' association times. By the end of slot $T$, user $i$ accrues a cumulative service $\sum_{j=1}^{T} \alpha_{i j} r_{i j}$. Hence, we formulate the following problem where the objective is to maximize a proportional fair cost function. ${ }^{8}$

Finite-horizon Proportional Fair (FPF) Scheduling:

$$
\begin{aligned}
\max _{\alpha} & C=\sum_{i=1}^{K} \log \left(\sum_{j=1}^{T} \alpha_{i j} r_{i j}\right) \\
\text { subject to } & \sum_{i=1}^{K} \alpha_{i j}=1 \quad \forall j=1 \ldots T \\
& \alpha_{i j} \in\{0,1\} .
\end{aligned}
$$

Even with full knowledge of $\mathbf{R}$, this problem is NP-hard (we omit the proof, see [20]). In practice, this problem has to be solved in an online (causal) manner. Users are scheduled slot-by-slot, based only on knowledge of the history and without full knowledge of $\mathbf{R}$. While the objective in the FPF Scheduling Problem is to maximize the proportional fairness metric (1), when evaluating the framework (Section VIII), we also consider the following metrics.

Definition 1 (Throughput): The average data rate allocated to all users, $\sum_{i=1}^{K} \sum_{j=1}^{T} \alpha_{i j} r_{i j} / T$ is referred to as throughput.

Definition 2 (Delay): The number of consecutive time slots in which a user $i$ does not receive an allocation is referred to as the delay and is denoted $d_{i}$. User $i$ is starved if $d_{i} \geq D_{\text {starved }}$, where $D_{\text {starved }}$ is a delay threshold.

We note that in Section VIII, the time horizon is sometimes considered in seconds, and is denoted by $\tilde{T}^{9}{ }^{9}$

\section{PREDictive FPF SCHEdULING (PF) ${ }^{2} \mathrm{~S}$ FRAMEWORK}

In this section, we review the widely deployed PF scheduling algorithm and present an online scheduling framework for solving the FPF Scheduling problem which combines two components: (i) data rate predictions and (ii) an estimation of future channel allocations. The design of these components will be presented in Sections VI and VII, respectively. We first describe the PF scheduler deployed in 3G networks [13] which is used in later sections as a benchmark.

\footnotetext{
${ }^{7}$ While in practice the number of associated users varies with time, we focus on a specific time-period with a given number of users.

${ }^{8}$ Although we focus on proportional fairness, the general approach can be applied to other concave cost functions (e.g., the $\alpha$-fairness class).

${ }^{9}$ In HSDPA, which is the $3 \mathrm{G}$ technology used in our measurement campaign, the slot length is $2 \mathrm{~ms}$ and hence, $\tilde{T}=T \cdot 2 \mathrm{~ms}$.
}

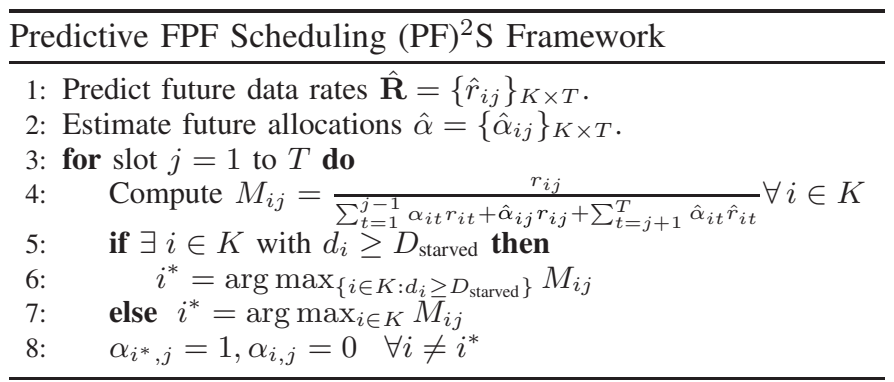

Definition 3 (PF-EXP [17], [25]): The scheduler which sets $\alpha_{i^{*} j}=1$ where $i^{*}=\arg \max _{i \in K} r_{i j} / R_{i}[j]$, and $R_{i}[j]=$ $(1-\epsilon) R_{i}[j-1]+\epsilon \alpha_{i j} r_{i j}$, is referred to as PF-EXP.

In the definition of the PF-EXP scheduler, $\epsilon$ determines the tradeoff between throughput and delay. With large values of $\epsilon(\approx 1)$, the scheduler puts more weight on the users' current feasible rates, thereby improving throughput at the expense of delay performance. With small values of $\epsilon(\approx 0)$ the users allocation history has more weight, and therefore, the delay performance improves at the expense of throughput. The PFEXP scheduler approaches optimal proportional fairness [17], [25] when the wireless channel state is a stationary process and users have long association times (i.e., $T \rightarrow \infty$ ).

Our Predictive FPF Scheduling $(\mathrm{PF})^{2} \mathrm{~S}$ Framework follows a similar approach as the PF-EXP scheduler to make slot-byslot allocations. It utilizes a gradient ascent approach [7] to maximize the objective function (1). In each time slot, the channel is allocated to the user corresponding to the largest objective function increase. Temporarily relaxing the integer constraints in (3), the gradient for user $i$ in time slot $j$ is:

$$
\frac{\partial C}{\partial \alpha_{i j}}=\frac{r_{i j}}{\sum_{t=1}^{T} \alpha_{i t} r_{i t}}=\frac{r_{i j}}{\sum_{t=1}^{j-1} \alpha_{i t} r_{i t}+\alpha_{i j} r_{i j}+\sum_{t=j+1}^{T} \alpha_{i t} r_{i t}} .
$$

Computing the above gradient requires knowledge of the entire data rate matrix $\mathbf{R}$ and is not feasible for an online algorithm, which only has knowledge of the past. Hence, the denominator of (4) is broken up into three components (from left to right): past, present, and future. From the perspective of an online scheduler, the first two components are known in any time slot. To enable slot-by-slot scheduling, the future component of (4) is computed as part of the (PF) ${ }^{2} \mathrm{~S}$ Framework, which is described in pseudo-code above.

Predictions of future data rates $\left(r_{i j}\right)$ and estimates of future channel allocations $\left(\alpha_{i j}\right)$ are denoted by $\hat{r}_{i j}$ and $\hat{\alpha}_{i j}$, respectively, with matrix representations denoted by $\hat{\mathbf{R}}$ and $\hat{\boldsymbol{\alpha}}$. At time 0 , predictions of $\hat{\mathbf{R}}$ and $\hat{\boldsymbol{\alpha}}$ are pre-computed for the entire horizon (next $T$ slots). These matrices can be generated using the methods described in Sections VI and VII but the framework can support other methods. For each user $i$ in each slot $j$, a ranking $M_{i j}$ which corresponds to (4), is computed using $\hat{\mathbf{R}}$ and $\hat{\boldsymbol{\alpha}}$. The user with the highest ranking is selected.

For a stationary channel, the future channel statistics are captured in the past component of the denominator (4). Hence, algorithms that rely only on past information (i.e., PF-EXP) are optimal. However, for non-stationary channel distributions, this does not hold. Hence, unlike in PF-EXP, step 4 in the framework considers the future channel component. 
TABLE I. SUMMARY OF COLLECTED MEASUREMENTS

\begin{tabular}{||l|c|c|c|c|c|c|c|c||}
\hline \hline Label & $\begin{array}{c}\text { Num } \\
\text { Logs }\end{array}$ & $\begin{array}{c}\text { Time } \\
\begin{array}{c}\text { Logged } \\
(\mathrm{min})\end{array}\end{array}$ & $\begin{array}{c}\text { Total } \\
\text { Dist. } \\
(\mathrm{km})\end{array}$ & $\begin{array}{c}\text { Av. Dist. } \\
(\mathrm{km})\end{array}$ & $\begin{array}{c}\text { Av. Velocity } \\
(\mathrm{m} / \mathrm{s})\end{array}$ & Total Sectors & $\begin{array}{c}\text { Total Serving } \\
\text { Sectors }\end{array}$ & $\begin{array}{c}\text { Total } \\
\text { Data } \\
(\mathrm{MB})\end{array}$ \\
\hline \hline $\mathrm{R} 1$ & 7 & 305.4 & 205.5 & 29.4 & 6.6 & 282 & 67 & 246 \\
\hline $\mathrm{R} 2$ & 4 & 85.2 & 33.0 & 8.2 & 10.7 & 245 & 42 & 254 \\
\hline R3 & 6 & 252.4 & 220.8 & 36.8 & 21.9 & 210 & 68 & 251 \\
\hline R4 & 10 & 339.5 & 351.0 & 35.1 & 16.8 & 963 & 336 & 1538 \\
\hline Static & 5 & 383.4 & - & - & - & 58 & 9 & 895 \\
\hline \hline Total & 32 & 1386.1 & 810.8 & - & - & 1758 & 522 & 3184 \\
\hline
\end{tabular}

By incorporating the predicted future, the $(\mathrm{PF})^{2} \mathrm{~S}$ Framework can leverage slow-fading trends. In addition, by making slotby-slot decisions, the framework also leverages fast-fading components, similar to PF-EXP.

Since the $(\mathrm{PF})^{2} \mathrm{~S}$ Framework aims to schedule users during slow-fading peaks (which may occur at several second intervals), it is essential to ensure that this does not result in long delays. Hence, in each slot, the framework first considers the set of starved users whose wait time $d_{i}$ (from the last slot of service) exceeds $D_{\text {starved }}$ (defined in Defn. 2) and selects one. If no user is starved, it selects among all users. Thereby, the framework can handle delay constraints. Note that delay considerations can be ignored by setting $D_{\text {starved }}=\infty$.

\section{Slow-Fading Measurements}

We now describe the measurements collected from a $3 \mathrm{G}$ network. Our analysis demonstrates that mobile users experience pronounced and reproducible slow-fading. The observation regarding reproducibility provides insights into the design of the data rate prediction $(\hat{\mathbf{R}})$ mechanism (Section VI).

\section{A. Measurement Setup and Test Drives}

The measurement campaign was conducted with Samsung Galaxy S II (GSII) Skyrocket phones [23]. The phone was connected via USB to a laptop running the Qualcomm eXtensible Diagnostic Monitor (QXDM) software. QXDM queries the phone in real-time and captures various physical layer attributes (described below) as well as GPS reports of location and velocity. QXDM records these measurements every $20 \mathrm{~ms}$, capturing the fast-fading and slow-fading components.

For the mobile measurements, the setup was placed in a car which traversed 4 different routes that span both highways and suburban roads (see Table I). For control purposes, we also performed measurements with a static (immobile) setup. During the measurements, a continuous download was conducted to ensure a sustained network connection. In summary, we measured wireless channel attributes during drives spanning $810 \mathrm{~km}$ and during a period of over 1,300 minutes. Further details of the measurements appear in [20].

\section{B. Channel State Metrics and Dynamics}

QXDM stores three physical layer attributes: the total inband power (including interference and noise), termed RSSI, the received pilot-power (RSCP), and the ratio between the pilot power and the total interference $\left(E_{c} / I_{o}\right){ }^{10}$ These key attributes characterize the channel quality and are periodically reported by the user to the serving sector [13]. While the latter two are specific to each nearby sector's pilot channel, the former (RSSI) is not. Moreover, while RSSI was commonly logged and used in previous work (e.g., [24]), from a scheduling perspective, $E_{c} / I_{o}$ is the most relevant indicator of a user's channel quality [13].

\footnotetext{
${ }^{10} E_{c} / I_{o}(d B)=R S C P(d B)-R S S I(d B)$.
}

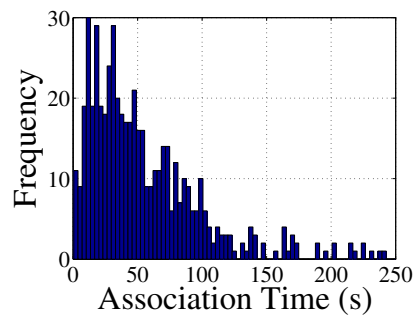

(a)

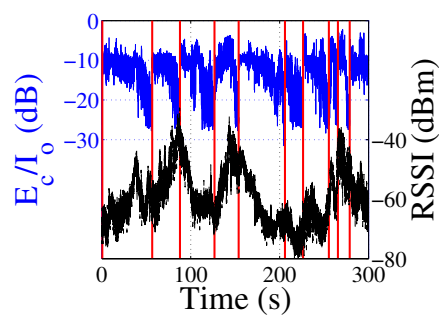

(b)
Fig. 2. (a) Distribution of sector association times for 27 drives along routes R1-R4 and (b) measured values of the RSSI and the serving sector $E_{c} / I_{o}$ for a drive on part of route R4 (vertical bars indicate hand-offs).

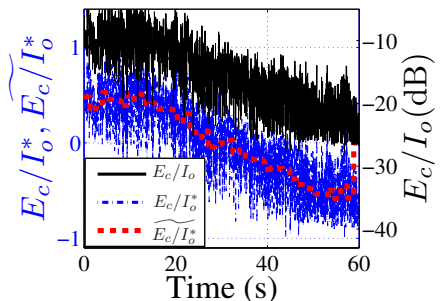

(a) Mobile Trace

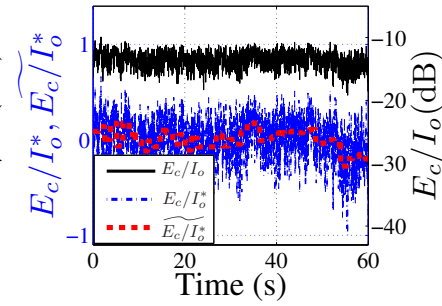

(b) Static (Immobile) Trace
Fig. 3. Comparison of $E_{c} / I_{o}$ (measured), $E_{c} / I_{o}^{*}$ (normalized), and $E_{c} / I_{o}^{*}$ (smoothed): (a) a mobile trace from route $\mathrm{R} 4$ and (b) a static trace.

We highlight the slow-fading phenomenon with an example. Fig. 2(a) shows a histogram of the users' association times for 27 drives on all routes, demonstrating that association times are on the order of tens of seconds. As a specific example, Fig. 2(b) shows measured traces of the RSSI and the serving sector $E_{c} / I_{o}$ for part of a single drive along route R4. Clearly, RSSI does not always reflect the same trend as $E_{c} / I_{o}$. Additionally, the $E_{c} / I_{o}$ experiences slow-fading on the order of several seconds. Since in most cases, the user's association times are tens of seconds, the slow-fading peaks and troughs occur within each sector. Therefore, we focus in the next two subsections on $E_{c} / I_{o}$ slow-fading trends, which are leveraged by the $(\mathrm{PF})^{2} \mathrm{~S}$ Framework.

\section{Slow-Fading and Mobility}

We first demonstrate that the slow-fading phenomenon is closely tied to user mobility. We then characterize the correlation between slow-fading and mobility metrics and show (in contrast to assumptions in past work, e.g., [2]) that slow-fading trends cannot be tied to simple line-of-sight metrics.

To quantify the slow-fading in a user's $E_{c} / I_{o}$ trace of $T$ slots, we define a slow-fading metric as described below. First, the mean is removed and the trace is normalized to obtain:

$$
E_{c} / I_{o}^{*}[j]=\frac{E_{c} / I_{o}[j]-\overline{E_{c} / I_{o}}}{\max _{1 \leq j \leq T}\left|E_{c} / I_{o}[j]-\overline{E_{c} / I_{o}}\right|} .
$$

The operation does not affect $E_{c} / I_{o}$ trends, but removes the amplitude which can vary depending on the sector, thereby enabling a comparison of $E_{c} / I_{o}$ traces from different sectors. Then, $E_{c} / I_{o}^{*}$ is smoothed by using wavelet transforms to remove the fast-fading components (with frequencies greater than $1 \mathrm{~Hz})$. The smoothed version of $E_{c} / I_{o}^{*}$ is denoted by $\widetilde{E_{c} / I_{o}^{*}}$ (details regarding the smoothing operation appear in [20]). Fig. 3 provides visual examples of $E_{c} / I_{o}^{*}$ and $\widetilde{E_{c} / I_{o}^{*}}$ for a mobile user and a static user. Using $\widehat{E_{c} / I_{o}^{*}}$ clearly illustrates 


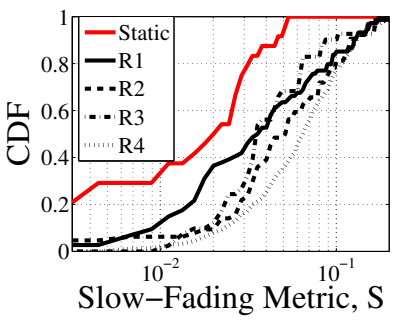

(a)

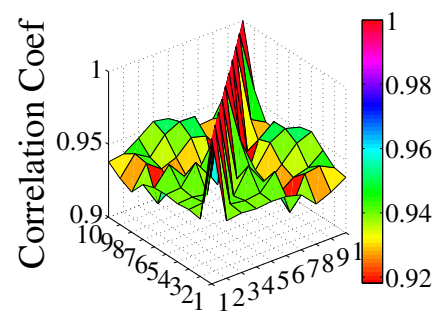

(b)
Fig. 4. (a) Characterization of slow-fading using the CDF of the slow-fading metric $(S)$ for mobile and static traces. (b) Correlation coefficients of measured $E_{c} / I_{O}$ values across 10 drives on route R4.

the presence (absence) of a trend in the values of $E_{c} / I_{o}$ over the time-period of observation.

Finally, we define the slow-fading metric as $S\left(\widetilde{E_{c} / I_{o}^{*}}\right)=$ $\sum_{j=1}^{T} \widehat{E_{c} / I_{o}^{*}}[j]^{2} / T . E_{c} / I_{o}$ traces with no appreciable trends will have $\widetilde{E_{c} / I_{o}^{*}}$ values close to zero (Fig. 3(b)) and hence $S$ will be small. On the other hand, $E_{c} / I_{o}$ traces with noticeable trends (Fig. 3(a)) will have large values of $\widetilde{E_{c} / I_{o}^{*}}$ (positive or negative) and hence larger values of $S$. As it is normalized by $T, S$ is used to compare $E_{c} / I_{o}$ traces with varying association times from different sectors.

We computed $S$ for every $E_{c} / I_{o}$ trace collected from every serving sector (see Table I). Fig. 4(a) shows the Cumulative Distribution Function (CDF) of the slow-fading metric values for routes R1-R4, as well as for the static traces. The mobile routes have much larger values of the slow-fading metric, confirming empirically that $S$ accurately distinguishes mobile traces with slow-fading from immobile traces.

Previous work assumed a strong correlation between slowfading and line-of-sight parameters (i.e., distance or velocity) [2], [9], supporting a functional prediction of the channel quality. However, our analysis indicates weak correlation between slow-fading and line-of-sight metrics. For example, in our traces, the correlation coefficient between $S$ and the distance or velocity is less than 0.2 (for more details, see [20]). Instead, slow-fading is governed by factors such as handoffs, landscape, and movement-induced shadowing, which are complex to model even in controlled scenarios [26].

\section{Slow-Fading Reproducibility}

As described above, the slow-fading trend is not directly associated with line-of-sight factors, and therefore, simple functional predictions are infeasible. Yet, the slow-fading component of $E_{c} / I_{o}$ is remarkably reproducible, enabling a datadriven prediction approach. Specifically, we observed that the $E_{c} / I_{o}$ from multiple measurements (from separate drives) is predictable with an error of $1-3 \mathrm{~dB}$ (a similar result appears in [24] for RSSI). To illustrate the reproducibility, we divide part of route R4 into $25 \mathrm{~m}$ segments ${ }^{11}$ and show in Fig. 1(b) the $E_{c} / I_{o}$ observed across a subset of segments for 3 of the drives on the route through 3 sectors. The overlap of the curves indicates the similarity across all drives.

We strengthen this observation by computing the crosscorrelation of $E_{c} / I_{O}$ across all drives for each route, as follows. Each route is divided into $25 \mathrm{~m}$ segments and each

\footnotetext{
$1125 \mathrm{~m}$ is the minimum guaranteed GPS resolution.
}

drive on this route is then represented by a vector of $E_{c} / I_{o}$ values, one for each segment (e.g., if a route includes $n$ segments, each drive is represented by a $n$-length vector, with multiple observations in the same segment represented by their average). We then compute the correlation coefficients of all the vectors (drives). Fig. 4(b) shows the correlation between 10 drives on route R4. Across all of the drives, the correlation coefficient is between 0.9-0.98 indicating a very high degree of correlation. Similar results are available in [20] for the other routes. The high correlation across all repeated drives implies that location-tagged historical measurements of $E_{c} / I_{o}$ can be used to accurately predict future slow-fading.

\section{Vi. Feasible Data Rate Prediction $(\hat{\mathbf{R}})$}

The $(\mathrm{PF})^{2} \mathrm{~S}$ Framework requires a mechanism to predict the users' feasible data rates for $T$ slots $(\hat{\mathbf{R}})$. We design such a mechanism, based on the observation that the slow-fading component of $E_{c} / I_{o}$ is highly reproducible, and refer to it as the Coverage Map Prediction Mechanism (CMPM). In an offline phase, measurement traces are processed to construct geographic coverage maps. The online phase is conducted by the sector and is composed of two steps. First, the user's location and velocity are determined. Then, this information is used in conjunction with the coverage map to predict user $i$ 's feasible rates $\hat{r}_{i j} \forall 1 \leq j \leq T$.

The first step can be implemented by querying the user's GPS. However, since this imposes energy and computation burdens on the user, we also develop the Channel History Localization Scheme (CHLS). The scheme assumes that knowledge of the user's overall trajectory exists. In Section VIII, we evaluate the framework using both alternatives.

\section{A. Coverage Map Construction}

The coverage map is constructed offline (once for each route) by placing a lattice over the geographic plane, and dividing it into square segments (see Fig. 5). Each segment, denoted by $b$, is covered by a set of sectors to which a user residing in it can associate, denoted by $U_{b}$. Cellular carriers routinely measure the channel quality on major routes. These measurements can be used to compute, for each segment $b$, an average RSSI value as well as average values of $E_{c} / I_{o}$ and RSCP for every nearby sector $u \in U_{b}$. These are denoted by $\overline{\operatorname{RSSI}}\langle b\rangle, \overline{E_{c} / I_{o_{u}}}\langle b\rangle, \overline{\operatorname{RSCP}_{u}}\langle b\rangle$. To compute these values for our evaluations, each sample measurement was tagged with a GPS location and tied to the appropriate segment.

\section{B. Channel History Localization Scheme (CHLS)}

The first step of the CMPM online phase localizes the user in the coverage map. To do this without GPS, we develop the CHLS. It matches the user's historical channel quality to coverage map segments on the user's trajectory, based on the differences between the channel metric values. Then, the user's location is estimated as the segment paired with its current channel quality value. Matching the channel quality history (i.e., a time-series) to segments (i.e., locations) depends on the user's velocity, which can vary. Hence, we utilize the Dynamic Time Warping (DTW) Algorithm ${ }^{12}$ to 'unwarp' the user's historical channel qualities to best fit the coverage map.

\footnotetext{
${ }^{12} \mathrm{~A}$ similar dynamic programming algorithm is used in speech recognition [21] to align two phrases which are offset (in time, amplitude, etc.).
} 


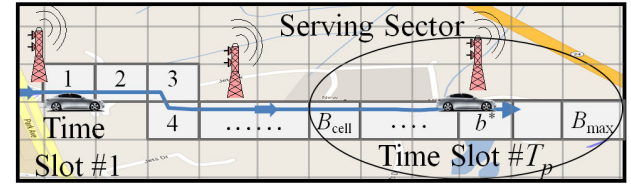

Fig. 5. Illustration of the CHLS: the coverage map segments are labeled starting from 1 (at time slot 1 , the user is in segment 1). At the present time slot $\left(T_{p}\right)$, the user is located in one of the segments between $B_{\text {cell }}$ and $B_{\max }$, which fall within the coverage area of the serving sector.

The CHLS requires knowledge of the user's trajectory and the user's location at a time slot in the recent history. ${ }^{13}$ The user's historical channel measurements are available at no extra cost as they are periodically reported to the network for scheduling and hand-off purposes.

The notation used to describe the scheme is defined below (see also Fig. 5). The sector keeps a history of the user's $E_{c} / I_{o_{u}}[j], \operatorname{RSCP}_{u}[j]$, and $\operatorname{RSSI}[j]$ for the past $T_{p}$ time slots, which are numbered sequentially from 1 to $T_{p}$ (present slot). The coverage map segments are sequentially numbered, starting with the segment which is the user's estimated location at slot 1 . The segments are numbered up to $B_{\max }$, which is the furthest segment in which the user could reside within the sector coverage area. The serving sector covers a range of segments $\mathbf{B}=\left\{b: B_{\text {cell }} \leq b \leq B_{\max }\right\}$.

The DTW Algorithm is applied to identify the cost of selecting each $b \in \mathbf{B}$ as the location estimate for the user. It constructs $H$, a matrix of size $B_{\max } \times T_{p}$. The value of entry $h_{b, j}$ represents the minimum cost of pairing time slots from 1 to $j$ with segments 1 to $b$. The constraint is that segment 1 is paired with slot 1 and segment $b$ is paired with slot $j$ (e.g., the end points are paired). The entries in the first row and column are, $h_{1, j}, h_{b, 1}=\infty \forall b, j$, and the rest of the matrix is computed using $h_{b, j}=\mathrm{c}(b, j)+\min \left(h_{b-1, j}, h_{b, j-1}, h_{b-1, j-1}\right)$, where the cost of matching segment $b$ to time slot $j$ is

$$
\begin{aligned}
& \mathrm{c}(b, j)=(\operatorname{RSSI}[j]-\overline{\operatorname{RSSI}}\langle b\rangle)^{2}+ \\
& \sum_{u \in U_{b}}\left(E_{c} / I_{o_{u}}[j]-\overline{E_{c} / I_{o_{u}}}\langle b\rangle\right)^{2}+\left(\operatorname{RSCP}_{u}[j]-\overline{\operatorname{RSCP}}_{u}\langle b\rangle\right)^{2} .
\end{aligned}
$$

If channel quality history does not exist for $u \in U_{b}$ at slot $j$, then $c(b, j)=\infty$. Note that the CHLS uses all three channel quality attributes to increase accuracy. Moreover, for each time slot, it utilizes channel quality attributes corresponding to several sectors. The scheme concludes by estimating that the user resides in $b^{*}=\operatorname{argmin}_{b \in \mathbf{B}} h_{b, T_{p}}$. To complete step one, the user's velocity is estimated, using training data to compute an average of past velocities near the estimated location.

The CHLS was evaluated via simulations. We set $T_{\mathrm{p}}=$ 3,000 slots (which corresponds to a horizon of $60 \mathrm{~s}$ ), set the segment size to $25 \mathrm{~m} \times 25 \mathrm{~m}$, and assumed that the serving sector coverage radius is $1,000 \mathrm{~m}$. We created coverage maps using half of the traces reported in Table I. From the remaining traces, we selected 500 random instances of 60 s-length. The distribution of localization errors is shown in Fig. 6(a). The scheme has a median error of $23 \mathrm{~m}$ and average error of $123 \mathrm{~m}$. For comparison, our evaluation of the RSSI-based localization scheme of [24] showed a median error of over $300 \mathrm{~m}$.

\footnotetext{
${ }^{13}$ For mobile users on highways and major roads, the trajectory can be estimated using mobility prediction techniques (e.g., [16]). The historical location can be reported based on past localization.
}

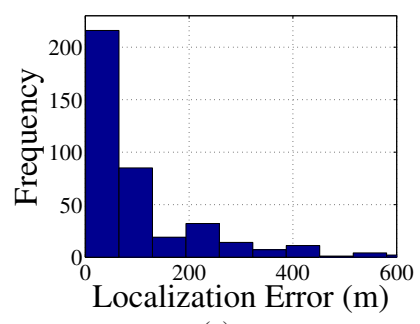

(a)

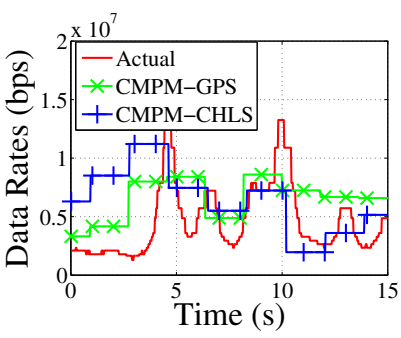

(b)
Fig. 6. Evaluation of the Coverage Map Prediction Mechanism (CMPM): (a) CHLS error distribution for 500 tests and (b) an example CMPM data rate prediction when location and velocity are determined using GPS or CHLS.

\section{Feasible Data Rate Prediction}

Recall that the FPF Scheduling Problem formulation is based on feasible data rates. Hence, we now transition to using data rates. The relation between $E_{c} / I_{o}$ and data rates (provided in [20]) is monotonic, and therefore, the reproducibility conclusions from Section V also apply to data rates.

A simple online algorithm that operates in the sector estimates the user's future data rates using a coverage map and an estimate of the user's current location and velocity (either from GPS or the CHLS). First, future locations are predicted assuming that the velocity is constant for future time slots. Each location is then mapped to a segment in the coverage map which in turn yields a data rate.

Fig. 6(b) shows an example data rate prediction for the CMPM using the two variations, to which we refer as CMPMGPS and CMPM-CHLS. In Section VIII, we demonstrate that the CMPM-CHLS captures enough of the slow-fading effects when integrated into the $(\mathrm{PF})^{2} \mathrm{~S}$ Framework to improve scheduling performance.

\section{Allocation Estimation $(\hat{\boldsymbol{\alpha}})$}

The $(\mathrm{PF})^{2} \mathrm{~S}$ Framework (described in Section IV) requires a channel allocation $(\hat{\boldsymbol{\alpha}})$ estimation algorithm based on the data rate predictions. This can be viewed as obtaining a solution to the FPF Scheduling Problem using the predicted data rate matrix $\hat{\mathbf{R}}$. As the framework operates in an online manner, the main design considerations are simplicity and robustness to prediction errors. We now introduce three algorithms which trade fairness and throughput performance for robustness to prediction errors. These algorithms will be evaluated with the rest of the framework in Section VIII.

Round Robin Estimation (RRE): This simple heuristic assumes that future time slots are allocated in a round-robin manner and each user receives an equal number of slots, resulting in an estimated allocation of $\hat{\alpha}_{i j}=1 / K \forall i, j$.

Blind Gradient Estimation (BGE): This heuristic utilizes (4) to select a user in each slot, but without the future component (since it is not known). Specifically, starting from $j=1$, it sets $\hat{\alpha}_{i^{*} j}=1$ where $i^{*}=\operatorname{argmax}_{i \in K}\left(\hat{r}_{i j}\right) / \sum_{t=1}^{j} \hat{\alpha}_{i t} \hat{r}_{i t}$. The expression contains only slot indices $\leq j$, and is similar to PF-EXP. BGE requires $O(K T)$ operations.

Local Search Estimation (LSE): This greedy algorithm, described below, initiates with an objective function value $C$ based on $\hat{\boldsymbol{\alpha}}$ composed of random values. It iterates slot-by-slot, greedily allocating slot $j$ to the user with the largest gradient 


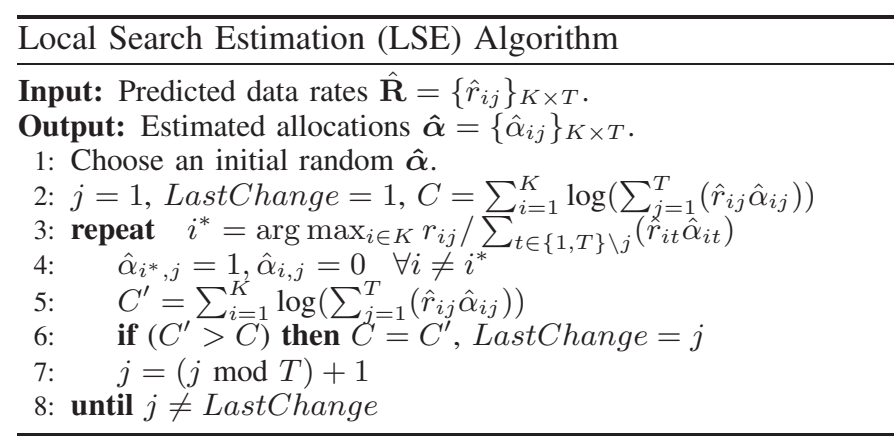

value, assuming all other time slots are fixed. The algorithm proceeds cyclically (returning to slot 1 after $T$ ) until reaching a local-maxima (i.e., no change in $T$ iterations). Termination is guaranteed as the objective value is bounded from above. Each cycle of LSE takes $O(K T)$ computations. Practically, it usually terminates after a few cycles.

An example of $\hat{\boldsymbol{\alpha}}$ values obtained by each of the algorithms appears in Fig. 7(a). LSE's estimates are tightly clustered near the predicted slow-fading peaks. The estimates from BGE are more diffused and those of RRE are uniform. Consequently, if the rate predictions are accurate, the framework using LSE provides the best performance, since it correctly allocates slots near the peak rates. The framework using BGE allocates slots around the peak rates, resulting in moderately good performance. The framework with RRE allocates slots uniformly, occasionally occurring during the peaks. On the other hand, if the prediction is erroneous, LSE would suffer, since it pushes the framework to schedule the user's slots at the predicted slow-fading peaks. BGE provides some robustness to prediction errors, and RRE is the most robust.

\section{PERFORMANCE EVALUATION}

We now use trace-based simulations to evaluate the performance of the $(\mathrm{PF})^{2} \mathrm{~S}$ Framework described in Section IV. Framework instances use combinations of CMPM implementations and channel allocation estimation algorithms. The test cases are generated using measurement traces and the performance metrics are proportional fairness (1) and throughput (Defn. 1). We show that various instances of our framework consistently outperform the deployed scheduler (PF-EXP), with throughput improvements in realistic scenarios ranging from $15 \%$ to $55 \%$. We then study the framework's sensitivity to the time horizon, number of users, mobility, $\hat{\mathbf{R}}$ accuracy, delay threshold, and coverage map resolution.

\section{A. Generation of Coverage Map and Test Cases}

From the dataset presented in Section V, half of the drives on each route were used as training measurements for coverage map construction (using a $25 \mathrm{~m} \times 25 \mathrm{~m}$ segment size). The remaining measurements were used for test case generation. A single test case was generated for every sector that had enough measurement data. Each test case is comprised of $K$ users and $T$ time slots and it emulates users starting at different locations within the sector coverage area and traveling with varying velocities in both directions along a route.

For each user $i$, the data rates $r_{i j}, 1 \leq j \leq T$ were generated by selecting a segment of $T$ random contiguous

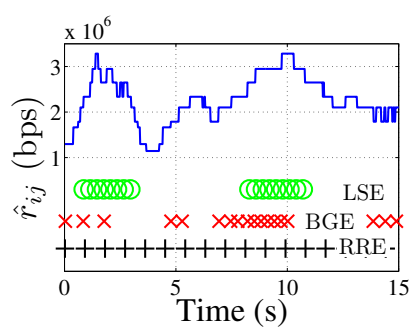

(a)

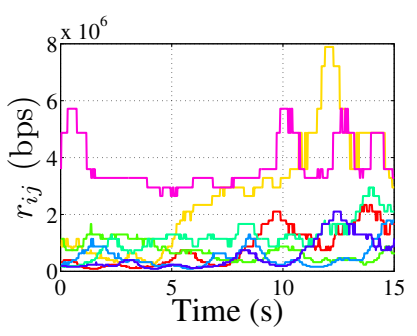

(b)
Fig. 7. (a) An example of predicted data rates for a user with $\tilde{T}=15 \mathrm{~s}$ and the corresponding $\hat{\alpha}$ estimations computed by the LSE, BGE, and RRE algorithms. (b) The data rates $\left(r_{i j}\right)$ for a test case with $K=7$ and $\tilde{T}=15$ s.

slots from part of the trace where the user was associated with the sector. In half of the cases, the vector was time-reversed, emulating travel in the opposite direction. Fig. 7(b) shows the data rates $\left(r_{i j}\right)$ for an example test case with $K=7$ and $\tilde{T}=30$ s (recall that $\tilde{T}$ is the time horizon in seconds).

Finally, for each generated rate matrix $\mathbf{R}$, we consider 3 approaches for obtaining the predicted rate matrix $\hat{\mathbf{R}}$ : clairvoyant (a.k.a., complete knowledge, $\hat{\mathbf{R}}=\mathbf{R}$ ), the CMPM which uses GPS information for location estimation (referred to as CMPM-GPS), and the CMPM which uses the CHLS (referred to as CMPM-CHLS). Using these approaches enables evaluating the framework with different qualities of $\hat{\mathbf{R}}$ prediction.

\section{B. Baseline Comparison and Upper Bound}

The $(\mathrm{PF})^{2} \mathrm{~S}$ Framework is compared to the deployed scheduler, PF-EXP (see Defn. 3), by normalizing the throughput and fairness values by the corresponding values obtained by PFEXP. Hence, metric values greater than 1 show improvements over PF-EXP. As an upperbound, the optimal solution to the FPF problem (referred to as OPT) is obtained using CVX, a MATLAB solver [1]. Note that OPT is obtained ignoring the integer constraints (3), using $\mathbf{R}$, and without delay constraints.

As mentioned in Section IV, the parameter $\epsilon$ implicitly controls the throughput-delay tradeoff for the PF-EXP scheduler. The $(\mathrm{PF})^{2} \mathrm{~S}$ Framework explicitly controls the throughputdelay tradeoff using the parameter $D_{\text {starved }}$. Unless otherwise specified, we fix $D_{\text {starved }}=0.5$ s (we discuss the sensitivity to this assumption in Section VIII-D). Correspondingly, based on extensive simulations, we set $\epsilon=0.01$ to provide similar delay performance as our framework, thus ensuring a fair comparison. See [20] for additional discussion on the throughput-delay tradeoff.

\section{Throughput and Fairness Gains}

We evaluate the throughput and fairness performance for various $(\mathrm{PF})^{2} \mathrm{~S}$ Framework instances and confirm experimentally that the $\hat{\alpha}$ estimation algorithms provide different degrees of robustness to rate prediction errors.

Figures 8(a) and (b) present box plots ${ }^{14}$ of the framework's fairness and throughput performance gains for 22 randomly generated test cases with $K=7$ and $\tilde{T}=30$ s (gains greater than 1 indicate improvements over PF-EXP). Since the objective function is logarithmic, the fairness gains are at the

\footnotetext{
${ }^{14}$ Box plots include a whisker at maximum and minimum samples, a box at the 25 th and 75 th sample quantile, and a line at the sample median.
} 


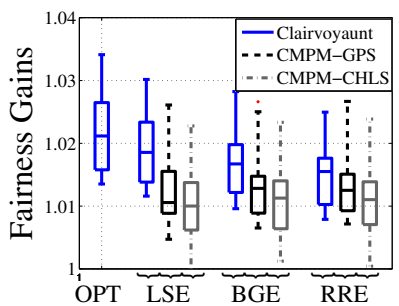

(a)

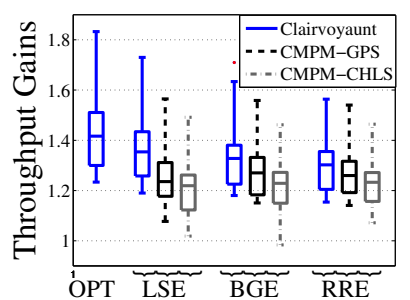

(b)

Fig. 8. $\quad(\mathrm{PF})^{2} \mathrm{~S}$ Framework performance for various framework instances (combinations of $\hat{\mathbf{R}}$ and $\hat{\alpha}$ prediction algorithms): statistical evaluation of 22 test cases with $K=7$ and $\tilde{T}=30 \mathrm{~s}$, and the resulting (a) fairness and (b) throughput gains over PF-EXP.

order of a few percent. The throughput gains over PF-EXP for all framework instances are significant (up to 70\%). Clearly, the performance of a framework instance depends on the rate prediction accuracy $(\hat{\mathbf{R}})$ and the channel allocation estimation $(\hat{\boldsymbol{\alpha}})$ algorithm. Hence, we consider framework instances, categorized by the $\hat{\mathbf{R}}$ prediction mechanism:

$\hat{\mathbf{R}}$ Clairvoyant: The throughput gains are substantial $(20 \%$ to $70 \%$ ). As expected, based on the framework instance performance, the estimation algorithms are ranked by LSE > BGE > RRE. In general, the LSE performance with complete knowledge was near optimal ${ }^{15}$ (an observation we justify analytically in [20]).

$\hat{\mathbf{R}}$ from CMPM-GPS: Fig. 8 shows that the ranking between the $\hat{\boldsymbol{\alpha}}$ estimation algorithms is BGE $>$ RRE $>$ LSE. As described in Section VII, BGE provides relative robustness to prediction errors, and hence with CMPM-GPS it often outperforms LSE with throughput gains of $20 \%$ to $55 \%$.

$\hat{\mathbf{R}}$ from CMPM-CHLS: The instances using LSE and BGE show the largest performance decrease (compared to using complete knowledge). Yet, they still result in gains over PFEXP. In general, we found that RRE is most resilient to errors and results in significant throughput gains of $15 \%$ to $50 \%$.

In summary, the evaluations with real-world measurements show that practical $(\mathrm{PF})^{2} \mathrm{~S}$ Framework instances consistently provide higher performance than the PF-EXP algorithm with throughput gains typically between $15 \%$ and $55 \%$.

\section{Sensitivity Analysis}

The results below are for the framework using RRE and CMPM-CHLS (similar results for other instances are in [20]).

Time Horizon $(\tilde{T})$ : Fig. 9(a) shows the fairness and throughput gains for test cases with varying time-horizons $\tilde{T}$. Intuitively, larger $\tilde{T}$ provides the framework additional opportunities to benefit over PF-EXP, which does not account for future data rates. For small to moderate values of $\tilde{T}(5 \mathrm{~s}, 15 \mathrm{~s})$, the framework shows $10 \%-30 \%$ throughput improvements. The performance gain for $\tilde{T}=60$ s increases to $20 \%-60 \%$. Eventually, as $\tilde{T}$ grows, the framework becomes limited by the accuracy of the prediction, which decays with time.

Number of Users (K): Fig. 9(b) shows the fairness and throughput gains for 20 test cases with $\tilde{T}=30$ s, and varying number of users. With additional mobile users, multi-

\footnotetext{
${ }^{15}$ For all test cases, when $D_{\text {starved }}=\infty$, the throughput when using LSE with complete knowledge is within $0.05 \%$ of the OPT throughput.
}

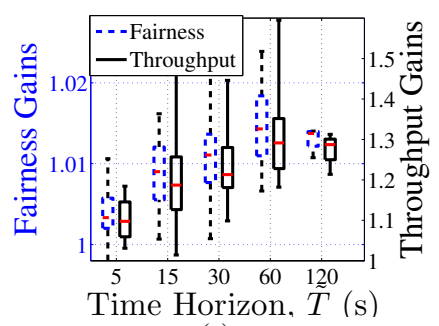

(a)

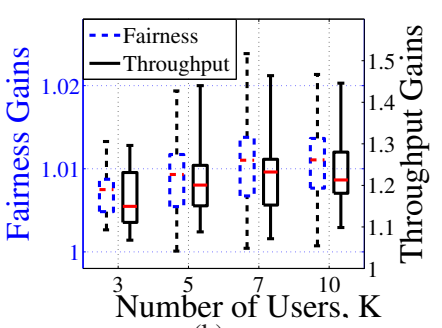

(b)
Fig. 9. (PF $)^{2} \mathrm{~S}$ Framework performance gains when using RRE with CMPMCHLS: statistical evaluation of (a) 10 test cases with $K=10$, varying the time horizon $(\tilde{T})$ and (b) 20 test cases of $\tilde{T}=30 \mathrm{~s}$, varying the number of users $(K)$.

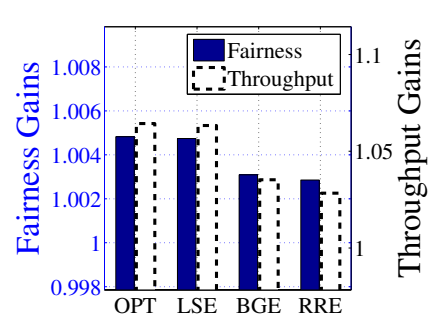

(a)

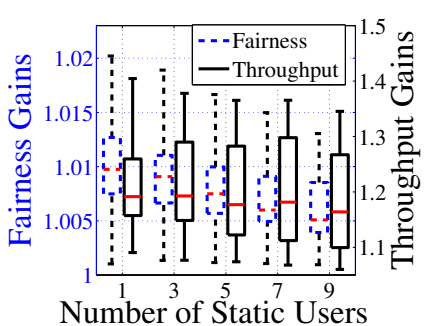

(b)
Fig. 10. $\quad(\mathrm{PF})^{2} \mathrm{~S}$ Framework performance gains: (a) a test case generated from static traces with $\tilde{T}=30$ s and $K=5$, and (b) statistical evaluation of 10 test cases using RRE with CMPM-CHLS with $\tilde{T}=30$ s, 10 mobile users, and varying number of static users.

user diversity increases and the performance improves. The throughput gains increase from up to $25 \%$ with 3 users to up to $45 \%$ with 10 users.

Effect of Mobility: To ascertain the affect of static users, we evaluate test cases created from the mobile and the static (immobile) measurements. With static measurements, the wireless channel state distribution is stationary. Fig. 10(a) considers the framework performance for all algorithms (with complete knowledge, as predictions are irrelevant in this case) in a test case with $K=5$ and $\tilde{T}=30$ s. The framework performance is very similar to PF-EXP (with throughput gains within 6\%) and is very close to OPT. Fig. 10(b) shows gains for 10 test cases of $\tilde{T}=30$ s with 10 mobile users and a varying number of static users. With the addition of static users, PF-EXP performance improves (approaches optimal), and therefore, the gains decrease. Yet, with 10 mobile users, the gains are still significant, with throughput gains of over $30 \%$ in some cases.

Slow-fading Peak Prediction: As indicated, the accuracy of the $\hat{\mathbf{R}}$ prediction impacts the framework performance. Through careful inspection, we found that a key factor in prediction accuracy is the location of the slow-fading peaks. Framework evaluations using a controlled $\hat{\mathbf{R}}$ prediction suggest that predicting the slow-fading peak within $5 \mathrm{~s}$ of the actual peak will result in significant performance improvements. Due to space constraints, these are provided in [20].

Delay Threshold ( $\left.D_{\text {starved }}\right)$ : It is important that, with the improved performance, the framework does not result in significant delay increases. The framework uses the delay threshold $D_{\text {starved }}$ to prioritize 'starved' users. For the example test case in Fig. 7(b), we vary $D_{\text {starved }}$ and observe the framework performance tradeoffs in Fig. 11(a). Tightening the delay threshold by an order of magnitude from $2 \mathrm{~s}$ to $0.25 \mathrm{~s}$ decreases the throughput gains from approximately $35 \%$ to 


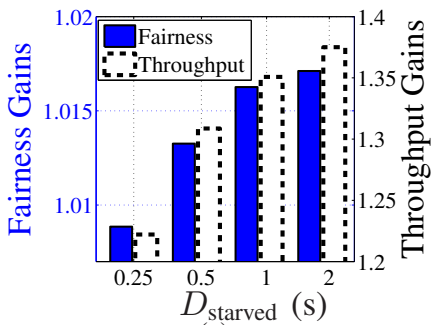

(a)

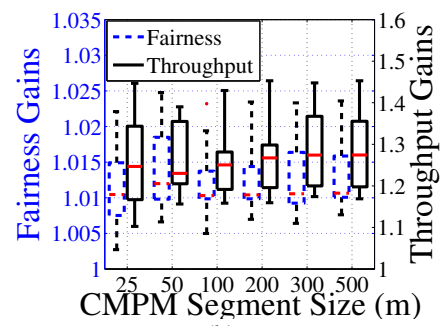

(b)
Fig. 11. $(\mathrm{PF})^{2} \mathrm{~S}$ Framework performance gains when using RRE with the CMPM-CHLS: (a) varying the delay threshold $D_{\text {starved }}$ for the test case given in Fig. 7(b), and (b) statistical evaluation of 15 test cases with $K=10$ and $\tilde{T}=30$ s for varying coverage map resolution values.

$25 \%$. This comparison was done with PF-EXP at a fixed value of $\epsilon=0.01$, which yields maximum delays of the order of $0.2-$ $0.4 \mathrm{~s}$. Hence, the framework provides similar delay performance along with higher throughput and fairness.

Coverage Map Resolution: In the results above, the coverage map segment size is $25 \mathrm{~m} \times 25 \mathrm{~m}$. Fig. 11(b) shows the framework gains for 15 test cases with $K=10$ and $\tilde{T}=30$ s as a function of the map segment size. The performance does not degrade significantly as the segment size becomes reasonably large, since larger segments result in averaging of channel quality attributes over a larger area. This indicates that coarse channel measurements are useful for the framework.

\section{COnclusions And Future Work}

We described an extensive wireless measurement study as well as the design and trace-based performance evaluation of the $(\mathrm{PF})^{2} \mathrm{~S}$ Framework. We showed that by leveraging slowfading, the framework (composed of various algorithms) can provide significant throughput gains while improving or maintaining fairness levels. Finally, we investigated the sensitivity of the results to different parameters and assumptions.

Future work will focus on relaxing the assumptions. Particularly, we plan to consider dynamic user populations handingoff between sectors. Additionally, we plan to extend the evaluations to consider policies that select appropriate $\hat{\alpha}$ estimation algorithms in different scenarios. Moreover, we will extend the localization scheme for cases in which trajectory information is unavailable or limited. Finally, as $4 \mathrm{G}$ networks become ubiquitous, we will conduct a corresponding measurement study and develop tailored resource allocation algorithms.

\section{ACKNOWLEDGEMENTS}

This work was supported in part by NSF grant CNS-1054856 and NSF CIAN ERC under grant EEC-0812072. We thank Howard Karloff for discussions regarding the solution framework.

\section{REFERENCES}

[1] CVX: Matlab Software for Disciplined Convex Programming, v2.0 beta

[2] S. H. Ali, V. Krishnamurthy, and V. C. Leung, "Optimal and approximate mobility-assisted opportunistic scheduling in cellular networks," IEEE Trans. Mobile Comput., vol. 6, no. 6, pp. 633-648, June 2007.

[3] J. Andersen, T. Rappaport, and S. Yoshida, "Propagation measurements and models for wireless communications channels," IEEE Commun. Mag., vol. 33, no. 1, pp. 42-49, 1995.

[4] M. Andrews and L. Zhang, "Scheduling over nonstationary wireless channels with finite rate sets," IEEE/ACM Trans. Networking, vol. 14 no. 5, pp. 1067-1077, Oct. 2006.
[5] M. Andrews, "A survey of scheduling theory in wireless data networks," in Wireless Communications, ser. The IMA Volumes in Mathematics and its Applications. Springer, 2007, vol. 143, pp. 1-17.

[6] H. Bang, T. Ekman, and D. Gesbert, "Channel predictive proportional fair scheduling," IEEE Trans. Wireless Commun., vol. 7, no. 2, pp. 482487, Feb. 2008.

[7] D. P. Bertsekas, Nonlinear Programming. Athena Scientific, 1999.

[8] S. Borst, N. Hegde, and A. Proutiere, "Mobility-driven scheduling in wireless networks," in Proc. IEEE INFOCOM'09, Apr. 2009.

[9] T. Bu, L. Li, and R. Ramjee, "Generalized proportional fair scheduling in third generation wireless data networks," in Proc. INFOCOM'06, 2006.

[10] G. Chandrasekaran, T. Vu, A. Varshavsky, M. Gruteser, R. Martin, J. Yang, and Y. Chen, "Tracking vehicular speed variations by warping mobile phone signal strengths," in Proc. IEEE PerCom'11, Mar. 2011.

[11] J. Hajipour and V. C. M. Leung, "Proportional fair scheduling in multicarrier networks using channel predictions," in Proc. IEEE ICC'10, May 2010.

[12] M. Hata, "Empirical formula for propagation loss in land mobile radio services," IEEE Trans. Veh. Technol., vol. 29, pp. 317-325, 1980.

[13] H. Holma and A. Toskala, Eds., WCDMA for UMTS: Radio Access for Third Generation Mobile Communications, 1st ed. John Wiley \& Sons, Inc., 2001.

[14] M. Ibrahim and M. Youssef, "CellSense: An accurate energy-efficient GSM positioning system," IEEE Trans Vehic. Techn., vol. 61, no. 1, pp. 286-296, Jan. 2012.

[15] F. Kelly, A. Maulloo, and D. Tan, "Rate control in communication networks: shadow prices, proportional fairness and stability," J. Oper. Res. Soc., vol. 49, pp. 237-252, 1998.

[16] J. Krumm and E. Horvitz, "Predestination: Where do you want to go today?" IEEE Computer, vol. 40, no. 4, pp. 105-107, 2007.

[17] H. Kushner and P. Whiting, "Convergence of proportional-fair sharing algorithms under general conditions," IEEE Trans. Wireless Commun., vol. 3, no. 4, pp. 1250-1259, July 2004.

[18] X. Liu, A. Sridharan, S. Machiraju, M. Seshadri, and H. Zang, "Experiences in a $3 \mathrm{G}$ network: interplay between the wireless channel and applications," in Proc. ACM MobiCom'08, Sept. 2008.

[19] X. Long and B. Sikdar, "A wavelet based long range signal strength prediction in wireless networks," in Proc. IEEE ICC'08, May 2008.

[20] R. Margolies, A. Sridharan, V. Aggarwal, R. Jana, N. Shankaranarayanan, V. Vaishampayan, and G. Zussman, "Exploiting mobility in proportional fair cellular scheduling: Measurements and algorithms," Columbia University, Tech. Rep. 2013-07-28, July 2013, available at http://wimnet.ee.columbia.edu/wp-content/uploads/2014/01/CUEE-2013-07-28.pdf.

[21] L. Rabiner and B.-H. Juang, Fundamentals of speech recognition. Prentice Hall, 1993.

[22] T. Rappaport, Wireless Communications: Principles and Practice, 2nd ed. Prentice Hall, 2001.

[23] Samsung Electronics Co., "Samsung Galaxy S II," http://www.samsung.com/global/microsite/galaxys $2 / \mathrm{html} / \mathrm{specification.html.}$

[24] A. Schulman, V. Navda, R. Ramjee, N. Spring, P. Deshpande, C. Grunewald, K. Jain, and V. N. Padmanabhan, "Bartendr: a practical approach to energy-aware cellular data scheduling," in Proc. ACM MobiCom'10, Sept. 2010.

[25] A. Stolyar, "On the asymptotic optimality of the gradient scheduling algorithm for multiuser throughput allocation," Operations Research, vol. 53, pp. 12-25, 2005.

[26] T. Su, H. Ling, and W. Vogel, "Markov modeling of slow fading in wireless mobile channels at $1.9 \mathrm{GHz}$," IEEE Trans. on Antennas and Propag., vol. 46, no. 6, pp. 947-948, June 1998.

[27] J. Yao, S. S. Kanhere, and M. Hassan, "An empirical study of bandwidth predictability in mobile computing," in Proc. ACM WiNTECH'08, Sept. 2008.

[28] —, "Geo-intelligent traffic scheduling for multi-homed on-board networks," in Proc. ACM MobiArch'09, June 2009. 